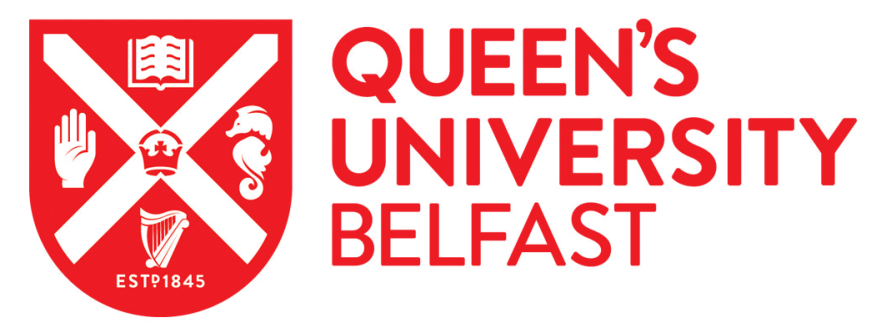

\title{
Cardiac 123I-MIBG normal uptake values are population-specific: Results from a cohort of controls over 60 years of age
}

Roberts, G., Lloyd, J. J., Kane, J. P. M., Durcan, R., Lawley, S., Howe, K., Petrides, G. S., O'Brien, J. T., \& Thomas, A. J. (2019). Cardiac 123I-MIBG normal uptake values are population-specific: Results from a cohort of controls over 60 years of age. Journal of nuclear cardiology : official publication of the American Society of Nuclear Cardiology. https://doi.org/10.1007/s12350-019-01887-6

Published in:

Journal of nuclear cardiology : official publication of the American Society of Nuclear Cardiology

\section{Document Version:}

Peer reviewed version

Queen's University Belfast - Research Portal:

Link to publication record in Queen's University Belfast Research Portal

\section{Publisher rights}

() American Society of Nuclear Cardiology 2019. This work is made available online in accordance with the publisher's policies. Please refer to any applicable terms of use of the publisher.

\section{General rights}

Copyright for the publications made accessible via the Queen's University Belfast Research Portal is retained by the author(s) and / or other copyright owners and it is a condition of accessing these publications that users recognise and abide by the legal requirements associated with these rights.

Take down policy

The Research Portal is Queen's institutional repository that provides access to Queen's research output. Every effort has been made to ensure that content in the Research Portal does not infringe any person's rights, or applicable UK laws. If you discover content in the Research Portal that you believe breaches copyright or violates any law, please contact openaccess@qub.ac.uk. 


\title{
Cardiac ${ }^{123}$ I-MIBG normal uptake values are population-specific: results from a cohort of controls over 60 years of age
}

\author{
G. Roberts ${ }^{1,2}$, J.J. Lloyd ${ }^{1,2}$, J.P.M. Kane ${ }^{1}$, R, Durcan' ${ }^{1}$, S. Lawley ${ }^{1}$, K. Howe ${ }^{2}$, G.S. Petrides ${ }^{2}$, J.T. \\ O'Brien $^{3}$, A.J. Thomas ${ }^{1}$
}

1: Institute of Neuroscience, Newcastle University, Biomedical Research Building, Campus for Ageing and Vitality, Westgate Road, Newcastle upon Tyne, UK. NE4 6BE

2: Nuclear Medicine Department, Leazes Wing, Royal Victoria Infirmary, Richardson Road, Newcastle upon Tyne, UK. NE1 4LP

3: Department of Psychiatry, University of Cambridge, Box 189, Level E4 Cambridge Biomedical Campus, Cambridge, CB2 OSP

Corresponding author:

Gemma Roberts

gemma.roberts@newcastle.ac.uk

ORCID ID: 0000-0002-6445-4023

\section{Running title:}

Cardiac MIBG uptake values are population-specific

\section{Acknowledgements}

We thank Edwin Poel and Dr. Derk Verschure (Amsterdam Medical Centre, Netherlands) and Prof. Kenichi Nakajima (Kanazawa University, Japan) for their kind assistance with gamma camera calibration and in particular Prof. Nakajima for further helpful discussions. We are extremely grateful to the people living with dementia and their families and the healthy older adults who participated in this study for giving up so much of their time to help dementia research.

\section{Funding}

Gemma Roberts is supported by an Alzheimer's Society healthcare professional Fellowship. Infrastructure and support was provided to authors based at Newcastle by the National Institute for Health Research (NIHR) Newcastle Biomedical Research Centre, a partnership between Newcastle upon Tyne Hospitals NHS Foundation Trust and Newcastle University. John O'Brien was supported by the NIHR Cambridge Biomedical Research Centre. GE Healthcare sponsored the visit of Edwin Poel for phantom calibration measurements. 


\section{Abstract}

Purpose

Cardiac I $^{123}$-MIBG image interpretation is affected by population differences and technical factors. We recruited older adults without cognitive decline and compared their cardiac MIBG uptake with results from the literature.

Methods

Phantom calibration confirmed that cardiac uptake results from Japan could be applied to our centre. We recruited 31 controls, 17 individuals with dementia with Lewy bodies (DLB) and 15 with Alzheimer's disease (AD). Images were acquired 20 minutes and four hours after injection using Siemens cameras with medium energy low penetration (MELP) collimators. Local normal heart-tomediastinum (HMR) ratios were compared to Japanese results.

Results

Siemens gamma cameras with MELP collimators should give HMRs very close to the calibrated values used in Japan. However, our cut-offs with controls were lower at 2.07 for early and 1.86 for delayed images. Applying our lower cut-off to the dementia patients may increase the specificity of cardiac MIBG imaging for DLB diagnosis in a UK population without reducing sensitivity.

Conclusions

Our local HMR cut-off values are lower than in Japan, higher than in a large US study but similar to those found in another UK centre. UK centres using other cameras and collimators may need to use different cut-offs to apply our results.

Keywords (4-6): Cardiac MIBG, HMR, Heart to mediastinum ratio, healthy controls, older adults 
Abbreviations

MIBG: Metaiodobenzylguanidine

FP-CIT: N- $\omega$-fluoropropyl- $2 \beta$-carbomethoxy-3 3 -(4-iodophenyl) nortropane

DLB: Dementia with Lewy bodies

AD: Alzheimer's Disease

HMR: Heart-to-mediastinum ratio

UK: United Kingdom

US: United states

LEHR: Low energy high resolution

MELP: medium energy low penetration 


\section{Background}

Dementia with Lewy bodies (DLB) is the second most common form of neurodegenerative dementia after Alzheimer's disease, accounting for 5-10\% of clinically diagnosed cases [1, 2]. Accurate diagnosis is important for clinical management, prognosis, and carer wellbeing [3-5], but initial misdiagnosis outside the specialist setting is common $[2,6,7]$. Schneider et al. found that about $15 \%$ of dementia cases at autopsy have Lewy bodies in the brain [8], further evidence that many people with DLB are being misdiagnosed during life.

Cardiac $^{123}$ I-metaiodobenzylguanidine (MIBG) sympathetic innervation imaging is now included as an indicative biomarker in the fourth DLB consensus criteria, alongside ${ }^{123}$ I-FP-CIT SPECT [6]. Cardiac MIBG studies are quantified on planar images using the heart-to-mediastinum ratio (HMR) as a diagnostic indicator, where HMR is the ratio between the count density in the cardiac left ventricle region (mean pixel value) and the count density in a mediastinum region of non-specific uptake. Abnormal scans have reduced cardiac uptake with a low HMR. Although the imaging technique is straightforward technically to implement in any hospital with a nuclear medicine service, the interpretation depends critically on establishment of a normal range, or cut-off for HMR values. We previously found that, provided that an appropriate normal HMR cut-off is used, HMR analysis is more accurate than visual rating [9]. The HMR normal range will depend on:

- Acquisition factors, including the camera and collimator

- Processing factors, such as region placement

- Population specific factors, for example obesity and heart disease

We addressed processing factors in our previous publication [9], with a standardised $6 \mathrm{~cm}$ circular cardiac ROI recommended. We discussed the effects of camera calibration and population specific factors $[9,10]$ and now address these here using images from older adults from a typical UK older population to generate a normal range. 
It has long been known that we need to standardise cardiac MIBG image acquisition and processing parameters in order for cardiac uptake thresholds to be applicable between centres, e.g. [11-16]. Medium energy collimators are recommended by the European Association for Nuclear Medicine for cardiac ${ }^{123}$ I-MIBG imaging as they give superior image quality for I-123, due to reduced septal penetration of high energy emissions [17]. However, LEHR collimators are usually used in the USA, probably as they were used in the large ADMIRE-HF study in heart failure [18].

Unfortunately, due to differences between vendors and collimator specification it is not always possible to generalise results from any medium energy collimator, as shown by the phantom studies of Nakajima et al. [19] and Verschure et al. [14]. Phantom calibration method can adjust for technical factors, for example collimator differences, but not for population differences.

In this study we aim to:

- Establish, for the first time, a normal HMR cut-off based on older adults without cognitive impairment in a typical UK population

- Compare the diagnostic accuracy of this UK cut-off for detecting Lewy body disease in a cohort of dementia patients to that of calibrated cut-off values from the literature.

\section{Methods}

\section{Phantom calibration}

Before starting cardiac I $^{123}$-MIBG imaging at our centre, a calibration check on our gamma cameras was performed with help from colleagues in Japan and the Netherlands, using a planar phantom. The method developed by Nakajima et al. $[11,19,20]$ was applied, which would allow us to see whether HMR cut-off values from Japanese studies needed to be modified for use locally. This calibration procedure revealed that the performance of our Siemens cameras fitted with medium energy low penetration (MELP) collimators was very similar to that of typical medium energy general purpose 
(MEGP) collimators in Japan (personal communication, Prof. K. Nakajima, Kanazawa University, Japan). Calibrated HMR cut-offs published by Komatsu et al. [21, 22] of 2.51 for early images and 2.20 for delayed images are therefore appropriate for use with our Siemens cameras with MELP collimators.

\section{Subjects}

Older controls without cognitive impairment:

Thirty-one individuals aged over 60 years with normal cognition were recruited as part of a Newcastle University study into the use of cardiac MIBG in a representative UK population of older adults. They underwent a detailed neurological examination by a research physician, including rating for parkinsonism with the Unified Parkinson's Disease Rating Scale (UPDRS), a thorough neurocognitive examination which confirmed they had normal cognition and an MRI brain scan reported by a radiologist as normal. Volunteers were excluded if they presented with cognitive impairment, major cerebrovascular disease on MRI brain imaging, Class II or worse heart failure according to the New York Heart Association classification, had had a myocardial infarction in the previous year, were taking labetalol or were unlikely to be able to tolerate imaging. Subjects were not excluded if they had risk factors for cardiac disease because we aimed to recruit typical older adults with normal cognition. Diabetes has been shown to be associated with reduced cardiac MIBG uptake in a small proportion of patients with clinical autonomic neuropathy [23] but similar to healthy controls in another study of patients with impaired glucose tolerance [24]. In our clinical studies in Lewy body disease we aim to assess cardiac MIBG in a "real-world" setting, where diabetes and risk factors for cardiac disease are extremely common. However, a detailed medical history including assessment of cardiac risk factors was taken.

Older people with dementia: 
Seventeen patients meeting the clinical criteria for probable dementia with Lewy bodies (DLB) and 15 meeting criteria for dementia due to Alzheimer's disease (AD) were recruited from memory clinics, as described in our previous publications $[9,10]$. Briefly, patients had their dementia diagnoses confirmed by an expert panel of old-age psychiatrists. They were categorised as having probable DLB if two or more of the core consensus criteria for DLB [6] (fluctuations, visual hallucinations, REM (rapid eye movement) sleep behaviour disorder and parkinsonism) were present, and AD if none of these were present. The same broad general inclusion criteria as discussed above for the controls, including being aged over 60 years, were applied to ensure the patients were typical of the local population.

\section{Image acquisition and processing}

Subjects were administered $111 \mathrm{MBq}$ I-123-MIBG via slow intravenous injection. Potassium iodate tablets $(170 \mathrm{mg}$ ) were given before and after injection to minimise uptake of free iodine by the thyroid. Ten minute anterior planar images were acquired at 20 minutes (early) and at 4 hours ( \pm 30 minutes) after injection (delayed). All images were acquired on a dual headed Siemens Symbia Intevo or Siemens Symbia gamma camera (Siemens Healthcare, Munich, Germany) with medium energy low penetration (MELP) collimators with energy window of $159 \mathrm{keV} \pm 10 \%$, matrix size $128 \times 128$ and no zoom applied.

All images were processed on a Hermes workstation (Hermes Medical Ltd, Stockholm). Planar images were analysed to obtain the heart to mediastinum ratio (HMR) using a $6 \mathrm{~cm}$ circular ROI placed over the left ventricle and $4 \times 3 \mathrm{~cm}$ rectangular ROI between the lungs in the mediastinum, as described in our previous publication [9]. Images were processed by a Medical Physics Expert (GR). Normal ranges based on control images were taken to be two standard deviations below the mean value and were calculated separately for early and delayed images. 


\section{Results}

\section{Control and patient demographics}

Of the 31 controls recruited, 2 were excluded due to their image appearance (see below). The remaining 29 people included were aged between 62 and 94 years (mean 75.2). Most were male $(22 / 29)$ and white (28/29). Body mass indices (BMI) ranged from 22 to $38 \mathrm{~kg} / \mathrm{m}^{2}$, with the mean value of 27.2 in the overweight category. The demographics for controls and patients are summarised in Table 1. The controls and dementia patients are taken from concurrent clinical studies, so not all the same information was collected for dementia patients.

Table 1: Demographics summary for the 29 controls, 17 DLB and 15 AD patients included in the study

\begin{tabular}{|c|c|c|c|}
\hline & Controls & DLB & AD \\
\hline $\begin{array}{l}\text { Age } \\
\text { Mean } \pm S D \\
\text { Range }\end{array}$ & $\begin{array}{l}75.2 \pm 8.3 \\
62 \text { to } 94 \text { years }\end{array}$ & $\begin{array}{l}77.5 \pm 8.0 \\
60 \text { to } 89 \text { years }\end{array}$ & $\begin{array}{l}76.2 \pm 6.8 \\
62 \text { to } 85 \text { years }\end{array}$ \\
\hline Sex & $\begin{array}{l}7 \text { female ( } 24 \%) \\
22 \text { male }(76 \%)\end{array}$ & $\begin{array}{l}2 \text { female (12\%) } \\
15 \text { male ( } 88 \%)\end{array}$ & $\begin{array}{l}4 \text { female }(27 \%) \\
11 \text { male }(73 \%)\end{array}$ \\
\hline $\begin{array}{l}\mathrm{BMI}\left(\mathrm{kg} / \mathrm{m}^{2}\right) \\
\text { Mean } \pm \mathrm{SD} \\
\text { Range }\end{array}$ & $\begin{array}{l}27.8 \pm 4.2 \\
21.6 \text { to } 37.8\end{array}$ & Not measured & Not measured \\
\hline Ethnicity & $\begin{array}{l}28 \text { white (97 \%) } \\
1 \text { Asian (3\%) }\end{array}$ & Not recorded & Not recorded \\
\hline Smoking & $\begin{array}{l}13 \text { never smoked (45 \%) } \\
13 \text { ex-smokers ( } 45 \%) \\
2 \text { current smokers }(7 \%) \\
1 \text { not recorded }(3 \%)\end{array}$ & Not measured & Not measured \\
\hline Diabetes & $\begin{array}{l}0 \text { Type I (0 \%) } \\
3 \text { Type II (10 \%) }\end{array}$ & Not recorded & Not recorded \\
\hline $\begin{array}{l}\text { Systolic blood } \\
\text { pressure } \\
\text { Mean } \pm S D \\
\text { Range }\end{array}$ & $\begin{array}{l}140.5 \pm 17.8 \\
112 \text { to } 173\end{array}$ & Not recorded & Not recorded \\
\hline $\begin{array}{l}\text { On blood } \\
\text { pressure } \\
\text { medication }\end{array}$ & $8(28 \%)$ & $4(24 \%)$ & $5(33 \%)$ \\
\hline
\end{tabular}




\begin{tabular}{|l|l|l|l|}
\hline $\begin{array}{l}\text { Hypercholesteri } \\
\text { naemia } \\
\text { documented }\end{array}$ & $11(34 \%)$ & Not recorded & Not recorded \\
\hline $\begin{array}{l}\text { Documented } \\
\text { cardiac history }\end{array}$ & $\begin{array}{l}\text { Previous MI and AF: } \\
1(3 \%) \\
\text { Angina: } 1(3 \%) \\
\text { ECG changes: } 1(3 \%)\end{array}$ & Previous MI: 2 (12\%) & Previous MI: 3 (20\%) \\
& & & \\
\hline
\end{tabular}

\section{Healthy control HMR results}

Of the 31 healthy older adults recruited, one had a markedly abnormal appearance on early and delayed cardiac MIBG imaging and one had an abnormal appearance on delayed imaging. The HMRs were also clearly reduced for these patients, shown as empty symbols on the plot in Figure 1. It is well recognised that occult Lewy body disease (usually termed incidental Lewy body disease) is present in many older people [25], which may explain these findings. These two control scans with abnormal appearance were therefore excluded from analysis to determine local normal HMR cut-offs. Figure 1 also shows the distribution of early and delayed control HMRs and the change in HMR between early and delayed.
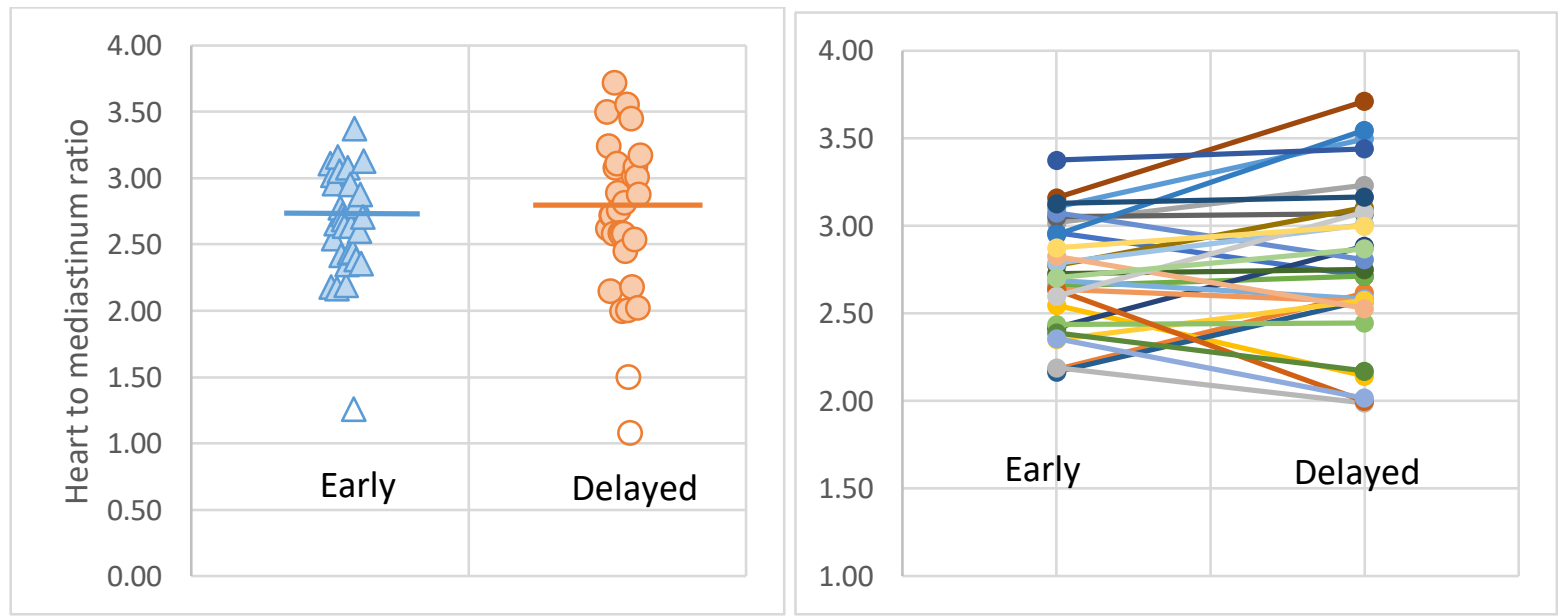

Figure 1: Left: early HMR values (blue triangles) and delayed (orange circles) with means of 2.72 (SD 0.32) and 2.78 (SD

0.46) respectively for the 29 included individuals. Excluded individuals are shown as empty symbols. Right: individual change in HMR between early (left) and delayed (right) images for each of the 29 controls. 
Eight of the 29 controls had HMR values below the Japanese cut-off of 2.51 on early images and 5/29 below 2.20 on delayed images.

Means, standard deviation and normal cut-offs (mean-2SD) derived from the control HMR data are shown in Table 2. The cut-off on the early images is higher due to the lower variation between individuals on the early images - the mean HMR is actually lower on the early images than delayed. With these cut-offs applied none of the 29 control subjects included were classified as abnormal.

Table 2: HMR data from healthy control subjects with normal scan appearances

\begin{tabular}{|l|l|l|l|l|}
\hline & $\mathrm{n}$ & Mean & Standard & Control \\
& & HMR & deviation & normal cut- \\
off
\end{tabular}

\section{Dementia patient results}

The mean HMR values for the patients with Alzheimer's disease and those with dementia with Lewy bodies from our previous work [10] are given in Table 3 with the control results included for comparison. Figure 2 shows plots of the HMRs for controls, AD patients and DLB patients for early and delayed images, with the cut-offs determined from controls shown as red lines and the Japanese cut-offs as purple lines. Three of the AD patients have uptake below the local cut-off (i.e. unexpectedly low) on early images and two on delayed. Six of the DLB patients have uptake above the local cut-off on early images, five of which are well above and 1 borderline $(H M R=2.15)$. This latter patient's HMR was 1.61 , clearly abnormal, on the delayed image. The other five patients retained a normal HMR on 
the delayed images, and the images appeared normal visually. The corresponding sensitivity, specificity and overall accuracy for DLB vs AD diagnosis using the local cut-offs are given in 
Table 4, alongside the results with the higher cut-off offs taken from the Komatsu et al. multicentre follow up study [22]. The third row shows the results with the optimal cut-offs for this cohort that we determined via ROC analysis in our previous work [10], which was 1.61 to 1.70 for delayed images. Similar analysis for early images gave the highest accuracy for cut-offs between 1.77 and 1.80 for early images.

Table 3: HMR results (mean and standard deviation) for DLB and AD patients.

\begin{tabular}{|l|c|c|c|c|c|}
\hline & & \multicolumn{2}{|c|}{ Early } & \multicolumn{2}{c|}{ Delayed } \\
\hline & $\mathrm{n}$ & mean & SD & mean & SD \\
\hline DLB & 17 & 1.88 & 0.71 & 1.69 & 0.79 \\
\hline $\mathrm{AD}$ & 15 & 2.47 & 0.41 & 2.48 & 0.41 \\
\hline Control & 29 & 2.72 & 0.32 & 2.78 & 0.46 \\
\hline
\end{tabular}

Early images

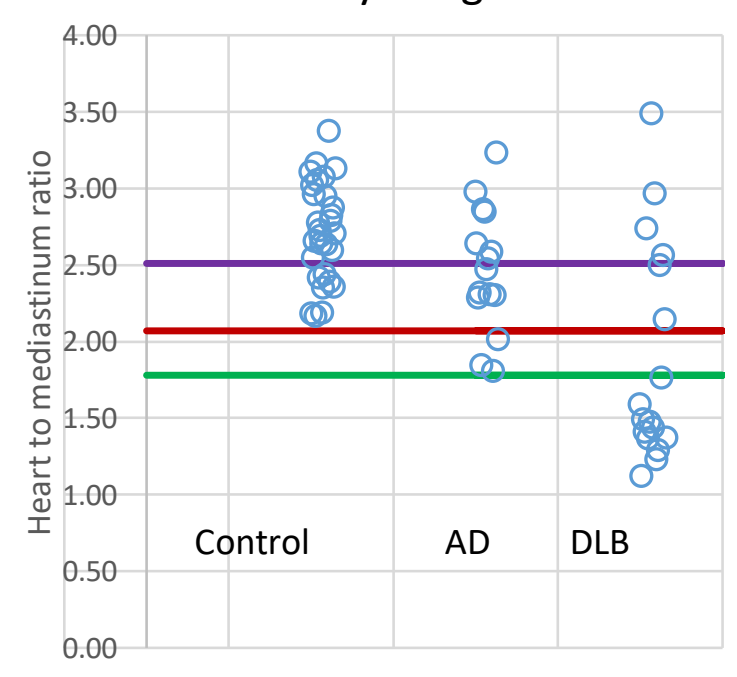

Delayed images

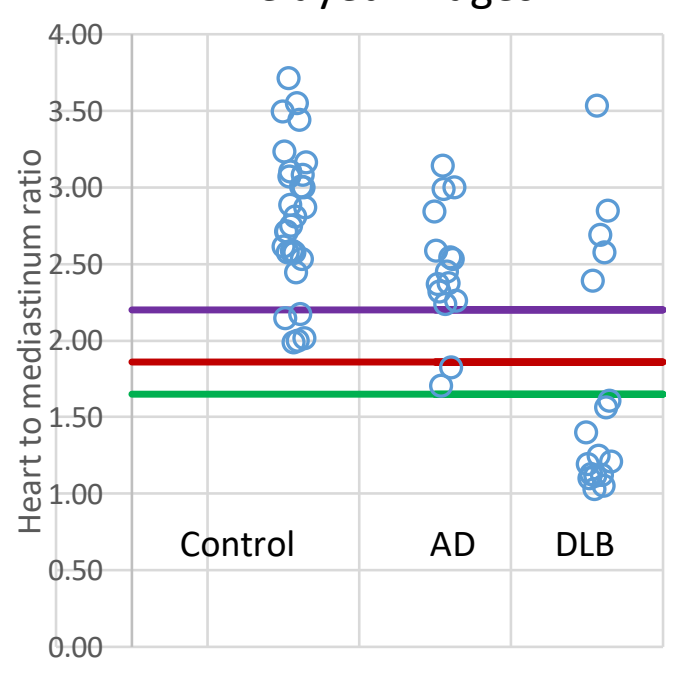

Figure 2: Heart to mediastinum ratios for controls, Alzheimer's disease and dementia with Lewy bodies patients for early images (left) and delayed images (right). The red lines show the local normal cut-off values, the green lines the optimal DLB vs $A D$ cut-offs for data from our previous study [10] and the purple lines the calibrated cut-off taken from the Japanese multicentre three year follow-up study [22]. 
Table 4: Sensitivity, specificity and overall accuracy for DLB vS AD differential diagnosis for early and delayed images with HMR cut-offs taken from local controls, Japanese multicentre study and ROC analysis of the data.

\begin{tabular}{|c|c|c|c|c|c|c|c|c|}
\hline \multirow{2}{*}{$\begin{array}{l}\text { Cut-off } \\
\text { source }\end{array}$} & \multirow{2}{*}{$\begin{array}{c}\text { Earl } \\
y\end{array}$} & \multirow{2}{*}{$\begin{array}{c}\text { Delaye } \\
\text { d }\end{array}$} & \multicolumn{3}{|c|}{ Early } & \multicolumn{3}{|c|}{ Delayed } \\
\hline & & & $\begin{array}{l}\text { Sensitivit } \\
\mathrm{y}\end{array}$ & $\begin{array}{l}\text { Specificit } \\
\text { y }\end{array}$ & $\begin{array}{l}\text { Accurac } \\
\mathrm{y}\end{array}$ & $\begin{array}{l}\text { Sensitivit } \\
\text { y }\end{array}$ & $\begin{array}{l}\text { Specificit } \\
\text { y }\end{array}$ & $\begin{array}{l}\text { Accurac } \\
\text { y }\end{array}$ \\
\hline $\begin{array}{l}\text { Local } \\
\text { controls }\end{array}$ & 2.07 & 1.86 & $65 \%$ & $80 \%$ & $72 \%$ & $71 \%$ & $87 \%$ & $78 \%$ \\
\hline $\begin{array}{l}\text { Komatsu et } \\
\text { al. (Japan) }\end{array}$ & 2.51 & 2.20 & $76 \%$ & $47 \%$ & $63 \%$ & $71 \%$ & $87 \%$ & $78 \%$ \\
\hline $\begin{array}{l}\text { Optimal } \\
\text { (ROC } \\
\text { analysis) }\end{array}$ & $\begin{array}{r}1.77 \\
\text { to } \\
1.80\end{array}$ & $\begin{array}{c}1.61 \text { to } \\
1.70\end{array}$ & $65 \%$ & $100 \%$ & $81 \%$ & $71 \%$ & $100 \%$ & $84 \%$ \\
\hline
\end{tabular}

\section{Discussion}

\section{Early and delayed cut-offs}

Eight of the 29 controls included in our study had HMR values below the early image cut-off of 2.51 from the Japanese follow-up DLB study [22] on early images and five of 29 below 2.20 on delayed images, providing strong evidence for lower thresholds for this population. We note that the value from the Japanese normal database (patient-based) gives a normal range of 2.2 to 4.0 for early images (mean 3.1) and 2.2 to 4.4 for delayed images (mean 3.3) [26]. These HMR ranges seen in Japanese patients without Lewy body disease do not seem to fit our UK population. If typical true HMR results vary between populations phantom calibration will not be sufficient, and measurements of local controls will be required. In our previous work we saw that when we applied a normal HMR cut-off derived from Japanese studies to our dementia patients it seemed much too high, with several Alzheimer's disease patients falling into the abnormal category [10]. Our cut-offs of 2.07 (early) and 
1.86 (delayed) reflect the higher variation in normal delayed HMR results compared with early results, rather than a difference in the mean, which is in line with previous studies [27-29]. These results were obtained using Siemens cameras fitted with MELP collimators, and as such cannot be directly applied to other centres using other collimators.

For early images, the use of the local control cut-off rather than the cut-off of Komatsu et al. (2.07 vs 2.51) resulted in two fewer DLB patients being correctly assigned as abnormal; one of whom had a less equivocal HMR on delayed imaging. The specificity was greatly increased with the local cut-off as only seven AD patients had early HMRs above the Japanese cut-off of 2.51. Use of the Japanese normal database result of 2.2 instead of 2.51 would not have affected either the sensitivity or specificity of early imaging. With delayed imaging, the sensitivity and specificity was the same with both the local cut-off and that of Komatsu et al. (1.86 vs 2.20$)$ as there were five DLB patients with clearly normal looking scans (lowest HMR $=2.39$ ) and there were no AD patients falling between the two cut-offs. It is worth noting however that five control subjects have HMR values between the two thresholds, suggesting our local values are more appropriate.

\section{Comparison with other studies}

At our centre we focus on the use of cardiac MIBG imaging for the diagnosis of Lewy body disease. However, as well as the differential diagnosis of Lewy body disease from other conditions [30-32], cardiac MIBG scintigraphy is also used for the estimation of risk of sudden cardiac death in heart failure patients and predicting those who could benefit from an implantable cardiac defibrillator (ICD) [18, 33] and this is currently the principal application in Europe and the US [34]. The largest study of healthy controls recruited to a cardiac MIBG research study ( $n=94)$ was that of Jacobsen et al. [35] carried out as part of the ADMIRE-HF heart failure study [18]. The controls were aged between 29 to 82 years (mean $58.5 \pm 10.6$ years, with BMl between 20.4 and 44.3 (mean $28.7 \pm 5.1$ ). These individuals were carefully screened to exclude coronary heart disease or Type 1 or 2 diabetes, making them healthier on average than the typical population. Although a substantial proportion (39/94) 
were aged over 60 years, the inclusion requirement of having an annual cardiac risk of less than $10 \%$ would further increase the difference in health between these individuals and a typical elderly population. We regard the inclusion of adults with diabetes and coronary risk factors as a strength of our study in relation to the diagnosis of Lewy body disease, rather than a weakness. In recruiting older adults that aside from having no cognitive impairment, are similar in general health to that of the general population we are determining a true normal range. However, despite this, the controls from the US ADMIRE study have lower HMR values on average than those from our study, even accounting for the different collimator. Using LEHR collimators their result was $1.78 \pm 0.22$, which would correspond to normal cut-off of 1.34 with LEHR (two standard deviations below the mean of 1.78 ). This cut-off converts to around 1.6 with our camera with MELP (determined using calibration data for LEHR collimators from [36]). One possible reason for the lower HMRs in the United States may be differences in the method used to delineate the cardiac ROI, with a freehand method used in the ADMIRE-HF study. We found previously that this tends to give lower HMR values than a $6 \mathrm{~cm}$ circular region [9]. Interestingly, the a priori cut-off used in the ADMIRE-HF study was much higher than subsequently seen in the controls: 1.6 with LEHR collimators, equivalent to around 2.1 for our camera with MELP (again, determined using calibration data for LEHR collimators from [36]). The a priori cutoff for the ADMIRE-HF study was determined by pooling the literature on cardiac MIBG values in healthy controls ( $n=202$ ) [37]. This was done without population or camera differences being taken into account, which may account for the discrepancy.

To our knowledge, the only other recent UK study in controls is by Asghar et al. in Manchester, again using LEHR collimators and excluding people any history of diabetes or cardiac disease [27]. In 14 healthy adults (mean age $54.6 \pm 5.4$ years, mean BMI $27.0 \pm 3.1$ ) they found a mean early HMR of 1.67 \pm 0.13 and delayed $1.73 \pm 0.16$, which would give cut-offs (mean - two standard deviations) of 1.41 for both early and delayed images. This cut-off is equivalent to around 1.7 for our camera with MELP collimation [36] and therefore similar to our result for delayed imaging of 1.86. Since there is thought to be little dependence of HMR on age $[38,39]$ we would expect any differences between our controls 
and this study to be due to the inclusion criteria, as it seems unlikely that there would be large differences between the population of Manchester and Newcastle, two post-industrial cities in northern England. The fact that our mean and cut-off values are slightly higher rather than lower than the Manchester study, after accounting for camera differences, provides further indirect support of our previous findings that cardiac disease is unlikely to have a big impact on HMR [10], and further supports the small body of evidence suggesting HMR is not strongly linked to age.

\section{Effect of cardiovascular disease on HMR}

It is known that regional cardiac sympathetic denervation assessed with SPECT or PET sympathetic imaging is associated with both areas of myocardial infarction (MI) and also with ischaemia in patients without previous MI [40-42]. The impact of $\mathrm{MI}$ and coronary artery disease (CAD) on global uptake assessed via planar cardiac MIBG scintigraphy is not well established, however most studies in healthy controls exclude those with CAD. To our knowledge there are no large studies that evaluate the impact of regional sympathetic denervation associated with MI and CAD on planar HMR. In this study and our previous work [10], one control and three AD patients had experienced an $\mathrm{MI}$, all more than 1 year prior to scanning. The control showed clear regional defects on SPECT imaging but borderlinenormal delayed planar HMR (2.14). The three AD cases showed defects of varying magnitude and extent on SPECT and planar HMRs of 2.99, 2.16 and 2.59. The numbers are too low to establish a correlation between the extent of regional denervation and effect on global HMR but it seems likely that regional denervation would have to be widespread for planar HMR to be abnormally low. Given the variation between healthy individuals, it is unlikely that MI and CAD alone would give an abnormal HMR result in individual cases. However it is likely that both the mean and standard deviation of a group of subjects would be affected by heart disease, suggesting that differences in the prevalence of cardiovascular disease between populations could contribute to differences in HMR cut-offs. In the UK the prevalence of cardiovascular diseases increases with age, with $8 \%$ of people between 60 and 70 years of age and $24 \%$ of those over 80 reporting at least one symptom [43]. In England the 
prevalence of coronary heart disease over all ages in 2018 was reported by the National Health Service to be $3.1 \%$ [44]. Equivalent data for Japan is not published but we note that a large cohort study (Akita-Osaka study) showed $0.2 \%$ incidence of new coronary heart disease cases in urban Japanese men aged between 60 and 69 between 1996 and 2003 [45] suggesting a much lower prevalence in this age group than in the UK.

\section{Effect of obesity on HMR}

Obesity has been linked to decreased apparent cardiac MIBG uptake by two previous studies $[38,46]$, and is thought to be due to increased attenuation and scatter in larger individuals, rather than to true differences in cardiac uptake. The ADMIRE control study discussed above [38] had a wide BMI range of 20.4 to $44.3 \mathrm{~kg} / \mathrm{m}^{2}$ (mean 28.7) and reported a "modest" decrease in HMR with BMI. This was a study into ageing and the incidental finding of a relationship between BMI and HMR was not discussed in detail. Pellegrino et al. reported lower HMRs in patients with BMl>30 $(n=10)$ compared to those with $\mathrm{BMI}<30(\mathrm{n}=35)[46]$. This was in heart failure patients and the results have not yet been replicated in healthy controls but do suggest patient size may affect apparent cardiac MIBG uptake. In our study the mean BMI of the controls was $27.8 \mathrm{~kg} / \mathrm{m}^{2}$ which is representative of the mean value of $27.3 \mathrm{for}$ 119,230 men aged 40-69 recruited into the UK biobank study between 2006 and 2010 [47]. The Manchester study had a similar mean BMI of 27.0. In contrast, the mean BMI in a Japanese longitudinal study of 104,928 individuals was 22.6 in men and 22.9 in women [48]. A relatively higher proportion of overweight individuals may therefore contribute to the lower mean HMRs in the UK and US compared to Japan. Future work using attenuation and scatter corrected SPECT imaging may provide insight into the contribution of patient size to these population differences in apparent cardiac MIBG uptake.

\section{Limitations}

This study was retrospective in nature, utilising control and patient data recruited to our centre for wider Lewy body disease studies. The inclusion of older adults with a range of cardiac risk factors and 
without detailed cardiac assessment to detect undiagnosed cardiovascular disease makes it difficult to determine the impact of cardiac disease on HMR. Very large samples of older adults with and without risk factors for cardiovascular disease could help to determine this, but as seen here, older people often have cardiac risk factors, making it impractical to recruit true healthy controls. As obesity is both a risk factor for cardiovascular disease and a potential cause of artefactually reduced HMR this would also need to be accounted for. If it were feasible, a large prospective study in multiple countries recruiting cognitively normal older adults typical of the local population and scanning them using the same protocol could determine whether HMR is population specific in a more rigorous manner.

\section{New Knowledge Gained}

We determined cardiac ${ }^{123}$ I-MIBG heart-to-mediastinum ratio cut-off values derived from a group of older adults in average health without evidence of cognitive decline for the first time. After accounting for camera differences, these values were lower than the values used in Japan, higher than in a large US study but similar to those found in another UK centre.

\section{Conclusion}

Our results suggest that normal cardiac ${ }^{123}$ I-MIBG heart-to-mediastinum ratio values may be population specific, although processing differences cannot be excluded. Further work is required to determine the reasons for these differences, which could include contributions from heart disease, obesity and other co-morbid conditions. This work highlights the importance of deriving HMR cut-offs from controls that are representative of the population being studied. 


\section{Compliance with ethical standards}

\section{Disclosure of potential conflicts of interest}

George Petrides and Gemma Roberts have received honoraria from GE Healthcare for educational presentations. Alan Thomas has received support from GE Healthcare for investigator led research. John O'Brien has acted as a consultant for GE Healthcare and received research support for investigator led research. Jim Lloyd, Joseph Kane, Rory Durcan, Sarah Lawley and Kim Howe declare that they have no conflict of interest.

\section{Ethical approval}

All procedures performed in studies involving human participants were in accordance with the ethical standards of the Newcastle University and Newcastle upon Tyne NHS Foundation Trust research committee and with the 1964 Helsinki declaration and its later amendments or comparable ethical standards. Informed consent was obtained from all individual participants included in the study.

\section{References}

1. Vann Jones, S.A. and J.T. O'Brien, The prevalence and incidence of dementia with Lewy bodies: a systematic review of population and clinical studies. Psychol Med, 2014. 44(4): p. 673-83.

2. $\quad$ Kane, J.P.M., et al., Clinical prevalence of Lewy body dementia. Alzheimers Res Ther, 2018. 10(1): p. 19.

3. Hanyu, H., et al., Differences in clinical course between dementia with Lewy bodies and Alzheimer's disease. Eur J Neurol, 2009. 16(2): p. 212-7.

4. Lee, D.R., et al., Examining carer stress in dementia: the role of subtype diagnosis and neuropsychiatric symptoms. Int J Geriatr Psychiatry, 2013. 28(2): p. 135-41.

5. Galvin, J.E., et al., Lewy body dementia: the caregiver experience of clinical care. Parkinsonism Relat Disord, 2010. 16(6): p. 388-92.

6. McKeith, I.G., et al., Diagnosis and management of dementia with Lewy bodies: Fourth consensus report of the DLB Consortium. Neurology, 2017. 89(1): p. 88-100.

7. Donaghy, P.C. and I.G. McKeith, The clinical characteristics of dementia with Lewy bodies and a consideration of prodromal diagnosis. Alzheimers Res Ther, 2014. 6(4): p. 46.

8. Schneider, J.A., et al., Cognitive impairment, decline and fluctuations in older communitydwelling subjects with Lewy bodies. Brain, 2012. 135(Pt 10): p. 3005-14. 
9. Roberts, G., et al., A comparison of visual and semiquantitative analysis methods for planar cardiac 123I-MIBG scintigraphy in dementia with Lewy bodies. Nucl Med Commun, 2019.

10. Kane, J.P.M., et al., (123)I-MIBG scintigraphy utility and cut-off value in a clinically representative dementia cohort. Parkinsonism Relat Disord, 2019.

11. Nakajima, K., et al., Standardization of 123I-meta-iodobenzylguanidine myocardial sympathetic activity imaging: phantom calibration and clinical applications. Clin Transl Imaging, 2017. 5(3): p. 255-263.

12. Verberne $\mathrm{HJ}$, et al., Vascular time-activity variation in patients undergoing ${ }^{123}$ I-MIBG myocardial scintigraphy: implications for quantification of cardiac and mediastinal uptake. Eur J Nucl Med Mol Imaging, 2011. 38(6): p. 7.

13. Verschure DO, et al., ${ }^{123}$ I-MIBG heart-to-mediastinum ratio is influenced by high-energy photon penetration of collimator septa from liver and lung activity. Nucl Med Commun, 2015. 36(3): p. 7.

14. Verschure, D.O., et al., A European myocardial 123I-mIBG cross-calibration phantom study. J Nucl Cardiol, 2017.

15. Slomka, P., et al., Quantification of I-123-meta-iodobenzylguanidine Heart-to-Mediastinum Ratios: Not So Simple After All. J Nucl Cardiol, 2014. 21: p. 979-983.

16. Klene, $\mathrm{C}$., et al., Influence of ROI definition on the heart-to-mediastinum ratio in planar 123/MIBG imaging. J Nucl Cardiol, 2016.

17. Flotats, A., et al., Proposal for standardization of 123I-metaiodobenzylguanidine (MIBG) cardiac sympathetic imaging by the EANM Cardiovascular Committee and the European Council of Nuclear Cardiology. Eur J Nucl Med Mol Imaging, 2010. 37(9): p. 1802-12.

18. Jacobson, A.F., et al., Myocardial iodine-123 meta-iodobenzylguanidine imaging and cardiac events in heart failure. Results of the prospective ADMIRE-HF (AdreView Myocardial Imaging for Risk Evaluation in Heart Failure) study. J Am Coll Cardiol, 2010. 55(20): p. 2212-21.

19. Nakajima, K., et al., Multicenter cross-calibration of I-123 metaiodobenzylguanidine heart-tomediastinum ratios to overcome camera-collimator variations. J Nucl Cardiol, 2014. 21(5): p. 970-8.

20. Nakajima, K., et al., Standardization of metaiodobenzylguanidine heart to mediastinum ratio using a calibration phantom: effects of correction on normal databases and a multicentre study. Eur J Nucl Med Mol Imaging, 2012. 39(1): p. 113-9.

21. Yoshita, M., et al., Diagnostic accuracy of 123I-meta-iodobenzylguanidine myocardial scintigraphy in dementia with Lewy bodies: a multicenter study. PLoS One, 2015. 10(3): p. e0120540.

22. Komatsu, J., et al., (123)I-MIBG myocardial scintigraphy for the diagnosis of DLB: a multicentre 3-year follow-up study. J Neurol Neurosurg Psychiatry, 2018.

23. Waqar, F. and M.C. Gerson, Cardiac sympathetic imaging in the diagnosis of cardiac autonomic neuropathy in pre-diabetes. J Nucl Cardiol, 2015. 22(6): p. 1269-72.

24. Asghar, O., et al., Individuals with impaired glucose tolerance demonstrate normal cardiac sympathetic innervation using I-123 mIBG scintigraphy. J Nucl Cardiol, 2015. 22(6): p. 12628.

25. Beach, T.G., et al., Multi-organ distribution of phosphorylated alpha-synuclein histopathology in subjects with Lewy body disorders. Acta Neuropathol, 2010. 119(6): p. 689-702.

26. Nakajima, K., et al., Normal values and standardization of parameters in nuclear cardiology: Japanese Society of Nuclear Medicine working group database. Ann Nucl Med, 2016. 30(3): p. 188-99.

27. Asghar, O., et al., lodine-123 metaiodobenzylguanidine scintigraphy for the assessment of cardiac sympathetic innervation and the relationship with cardiac autonomic function in healthy adults using standardized methods. Nucl Med Commun, 2017. 38(1): p. 44-50.

28. Yoshita, M., et al., Value of 123I-MIBG radioactivity in the differential diagnosis of DLB from $A D$. Neurology, 2006. 66(12): p. 1850-4. 
29. Sakamoto, F., et al., 123I-MIBG myocardial scintigraphy for the evaluation of Lewy body disease: are delayed images essential? Is visual assessment useful? $\mathrm{Br} J$ Radiol, 2016: $\mathrm{p}$. 20160144.

30. Chung, E.J. and S.J. Kim, (123)I-Metaiodobenzylguanidine Myocardial Scintigraphy in Lewy Body-Related Disorders: A Literature Review. J Mov Disord, 2015. 8(2): p. 55-66.

31. Orimo, S., et al., (123)I-meta-iodobenzylguanidine (MIBG) cardiac scintigraphy in alphasynucleinopathies. Ageing Res Rev, 2016. 30: p. 122-33.

32. Sonni, I., et al., Clinical validity of presynaptic dopaminergic imaging with (123)I-ioflupane and noradrenergic imaging with (123)I-MIBG in the differential diagnosis between Alzheimer's disease and dementia with Lewy bodies in the context of a structured 5-phase development framework. Neurobiol Aging, 2017. 52: p. 228-242.

33. Verberne, H.J., et al., Prognostic value of myocardial 123I-metaiodobenzylguanidine (MIBG) parameters in patients with heart failure: a systematic review. Eur Heart J, 2008. 29(9): p. 1147-59.

34. Travin, M.I., et al., How do we establish cardiac sympathetic nervous system imaging with (123)I-mIBG in clinical practice? Perspectives and lessons from Japan and the US. J Nucl Cardiol, 2018.

35. Jacobson, A.F., et al., Impact of age on myocardial uptake of (1)(2)(3)I-mIBG in older adult subjects without coronary heart disease. J Nucl Cardiol, 2013. 20(3): p. 406-14.

36. Nakajima, K., et al., The time has come to standardize (123)I-MIBG heart-to-mediastinum ratios including planar and SPECT methods. Eur J Nucl Med Mol Imaging, 2016. 43(2): p. 3868.

37. Jacobson, A.F., et al., 123I-mIBG scintigraphy to predict risk for adverse cardiac outcomes in heart failure patients: design of two prospective multicenter international trials. J Nucl Cardiol, 2009. 16(1): p. 113-21.

38. Jacobson AF, et al., Impact of age on myocardial uptake of 123I-mIBG in older adult subjects without coronary heart disease. . J Nucl Cardiol, 2013. 20: p. 9.

39. Nakajima, K., et al., Is (123)I-metaiodobenzylguanidine heart-to-mediastinum ratio dependent on age? From Japanese Society of Nuclear Medicine normal database. Ann Nucl Med, 2018. 32(3): p. 175-181.

40. Nakata, T., et al., Regional cardiac sympathetic nerve dysfunction and the diagnostic efficacy of metaiodobenzylguanidine tomography in stable coronary artery disease. Am J Cardiol, 1996. 78(3): p. 292-7.

41. Travin, M.I., Potential for adrenergic imaging to serve as a unique tool for guidance of patient management during and after an acute ischemic event. J Nucl Cardiol, 2018. 25(2): p. 581-585.

42. Fallavollita, J.A. and J.M. Canty, Jr., Dysinnervated but viable myocardium in ischemic heart disease. J Nucl Cardiol, 2010. 17(6): p. 1107-15.

43. Hinton, W., et al., Incidence and prevalence of cardiovascular disease in English primary care: a cross-sectional and follow-up study of the Royal College of General Practitioners (RCGP) Research and Surveillance Centre (RSC). BMJ Open, 2018. 8(8): p. e020282.

44. Digital, N., Quality and Outcomes Framework, Achievement, prevalence and exceptions data - 2017-18. Quality and Outcomes Framework (QOF), 2018.

45. Kitamura, A., et al., Trends in the incidence of coronary heart disease and stroke and their risk factors in Japan, 1964 to 2003: the Akita-Osaka study. J Am Coll Cardiol, 2008. 52(1): p. 71-9.

46. Pellegrino, T., et al., Impact of obesity and acquisition protocol on (123)Imetaiodobenzylguanidine indexes of cardiac sympathetic innervation. Quant Imaging Med Surg, 2015. 5(6): p. 822-8. 
47. Bradbury, K.E., et al., Association between physical activity and body fat percentage, with adjustment for BMI: a large cross-sectional analysis of UK Biobank. BMJ Open, 2017. 7(3): p. e011843.

48. Cui, R., et al., Body mass index and mortality from cardiovascular disease among Japanese men and women: the JACC study. Stroke, 2005. 36(7): p. 1377-82. 